\title{
Periostin regulates fibrocyte function to promote myofibroblast differentiation and lung fibrosis
}

\author{
SL Ashley ${ }^{1}$, CA Wilke ${ }^{2}, \mathrm{KK} \mathrm{Kim}{ }^{2}$ and BB Moore ${ }^{2,3}$
}

Fibrocytes are circulating mesenchymal precursors (CD45+, col 1+) recruited to fibrotic areas. Fibrocytes secrete profibrotic mediators including periostin; a matricellular protein that regulates cellular interactions with extracellular matrix $(E C M)$ components. In bleomycin-induced fibrosis, periostin deficiency in structural or hematopoietic cells limits development of pulmonary fibrosis. To determine if hematopoietic-derived fibrocytes might secrete soluble factors to activate structural myofibroblast differentiation, wild-type (WT) fibroblasts were treated with conditioned medium from fibrocytes isolated from bleomycin-treated WT or periostin ${ }^{-\prime}$ mice. After $24 \mathrm{~h}$ we saw less $\alpha$-smooth muscle actin expression in cells treated with conditioned medium from periostin ${ }^{-1-}$ fibrocytes. Adoptive transfer of WT fibrocytes augmented lung fibrosis to a greater extent than transfer of fibrocytes from periostin ${ }^{-/}$mice. In vitro analysis of fibrocytes and fibroblasts isolated from WT and periostin ${ }^{-1-}$ mice treated with TGF $\beta 1$ or periostin demonstrated co-regulation of mesenchymal activation and beta 1 integrin as a potential receptor for periostin on fibrocytes. Additionally, connective tissue growth factor (CTGF) mRNA expression was increased in fibrocytes treated with periostin whereas CTGF and lysl oxidase (LOX) mRNA expression was low in bleomycin-treated periostin ${ }^{-1-}$ fibrocytes. These data suggest fibrocytes may augment bleomycin-induced fibrosis via secretion of periostin and other soluble factors that promote myofibroblast differentiation.

\section{INTRODUCTION}

Fibrosis can be triggered by various known insults (e.g., infection, allergens, toxins, or radiation), or can occur for unknown reasons as in the case of idiopathic pulmonary fibrosis (IPF). ${ }^{1}$ IPF is a chronic progressive parenchymal lung disease of unknown origin, with mean survival rate of 3-5 years after diagnosis. Prevalence and mortality rates are increasing globally. ${ }^{2-4}$ The cellular mechanisms leading to IPF remain unclear but a commonly held paradigm describes continuous cycles of injury to the alveolar epithelium leading to dysregulated repair. Prominent features of this dysregulated repair include fibroblast differentiation, myofibroblast accumulation and excessive collagen deposition in the alveolar space. ${ }^{5-7}$ Mediators such as growth factors and cytokines from different cell types contribute to the persistence of activated fibroblasts and myofibroblasts. ${ }^{8}$ Based on our current knowledge, bone marrow-derived collagen producing cells (e.g. fibrocytes and other collagen producing cells) play a significant role in the development of lung fibrosis. ${ }^{9-17}$ Fibrocytes respond to a number of cytokines and chemokines that are typically associated with migration and activation of inflammatory cells. ${ }^{18}$ Fibrocytes are hematopoietic bone marrow-derived cells that express both mesenchymal and leukocyte markers. They circulate in the peripheral blood and can be isolated from tissues. Cultured fibrocytes have been shown to express a number of ECM proteins including collagen 1, collagen 3 and fibronectin. ${ }^{18-22}$ In addition, fibrocytes also express a number of chemokine receptors, including CXCR4, CCR7 and CCR2, which may contribute to the recruitment and activation of fibrocytes resulting in migration to damaged tissues. ${ }^{23-25}$ Although several reports indicate that fibrocytes express type 1 collagen, others have suggested that uptake of secreted type 1 collagen by hematopoietic cells characterizes the fibrocyte population. ${ }^{26,27}$

A more recent study demonstrated that cells of hematopoietic origin produce type 1 collagen but are not a necessary

${ }^{1}$ Graduate Program in Immunology, University of Michigan, Ann Arbor, Michigan, USA. ${ }^{2}$ Department of Internal Medicine, Division of Pulmonary and Critical Care Medicine, University of Michigan, Ann Arbor, Michigan, USA and ${ }^{3}$ Department of Microbiology and Immunology, University of Michigan, Ann Arbor, Michigan, USA. Correspondence: BB Moore (bmoore@umich.edu) 


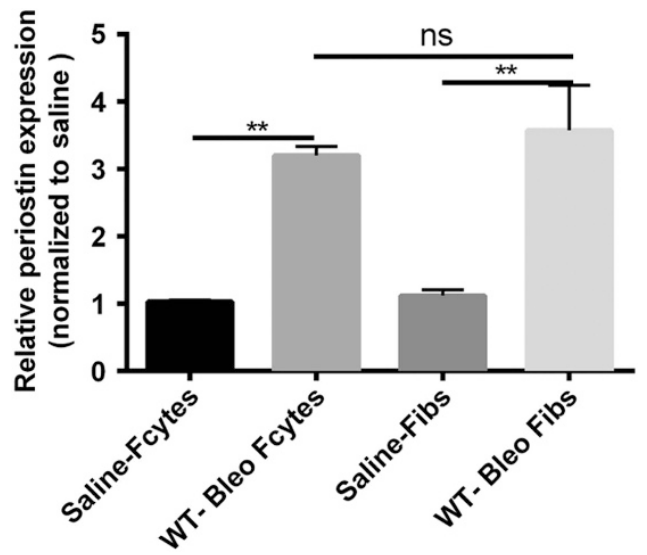

Figure 1 Increased mRNA expression of periostin in lung mesenchymal cells post-bleomycin treatment. Wild type mice were given $0.025 \mathrm{U}$ of bleomycin or saline intratracheally on day 0 . On day 14 , lung mesenchymal cells were cultured and sorted by magnetic bead separation for fibroblasts (fibs) and fibrocytes (fcytes). Total RNA was isolated and by real time RTPCR we measured the expression of periostin and $\beta$-actin in fibrocytes and fibroblasts. Data represent $n=3$ per group pooled from multiple mice. ${ }^{\star *} P<0.01,{ }^{\star} P<0.05$, ns $=$ not significant.

source of type 1 collagen during experimental lung fibrosis. ${ }^{28}$ Kleaveland et al. illustrated that fibrocytes can efficiently uptake type 1 collagen even though the underlying mechanism is not clearly understood. ${ }^{28}$ Adoptive transfer of fibrocytes leads to augmented fibrosis in a FITC-induced mouse model and several studies have found a correlation between increased numbers of fibrocytes and worse disease progression..$^{22,24,29-31}$ Recently, fibrocytes were shown to contribute to the progression of pulmonary fibrosis without differentiating to myofibroblasts themselves using a transforming growth factor alpha model of fibrosis. ${ }^{32}$ Additionally, Garcia de alba et al. also showed that fibrocytes contributed to increased mRNA levels of ECM proteins in both fibrosis and chronic pneumonitis. ${ }^{31}$ Work from our laboratory demonstrated that fibrocytes in circulation of IPF patients are major producers of the matricellular protein, periostin, and that periostin accumulates in the lung tissue of IPF patients. ${ }^{33}$

Periostin, also known as osteoblast-specific factor 2, is a recently characterized matricellular protein that binds to components of the ECM including type 1 collagen and fibronectin and has been shown to be involved in collagen fibrillogenesis. ${ }^{34}$ Periostin protein transmits signals from the ECM to the cell by binding to cellular receptors such as integrins that affect cell adhesion, proliferation, migration and tissue angiogenesis. ${ }^{35}$ It has been reported that periostin promotes cancer cell invasion and metastasis through the integrin/phosphatidylinositol 3-kinase/AKT pathway, leading to the development of various tumors. ${ }^{36}$ Work from our laboratory and others have shown that there are increased circulating periostin levels in IPF patients compared to controls. ${ }^{33,37}$ Our work further showed that the increased periostin expression in IPF tissues was localized to active areas of fibrosis and that IPF patients also showed increased percentages of periostin-expressing fibrocytes and monocytes in the blood. ${ }^{33}$ Chimeric mouse studies showed that both structural and hematopoietic sources of periostin were important in protecting periostin-deficient mice from bleomycin-induced fibrosis. ${ }^{33}$ Although periostin has diverse functions, it is becoming increasingly clear that periostin is upregulated in lung tissue in the context of several respiratory diseases. ${ }^{38}$ At present, the precise function of periostin in the lung has not been fully elucidated. In this study we used bleomycin to establish fibrosis in mice, and then analyzed how hematopoietic-derived fibrocytes might signal structural myofibroblast differentiation through their ability to secrete periostin or other periostin-regulated soluble factors. We explored the use of periostin-deficient fibrocytes in adoptive transfer experiments as well as the use of integrin blocking antibodies to demonstrate that periostin interacts with different receptors on fibrocytes and fibroblasts and that periostin secretion by fibrocytes is important for the exacerbation of bleomycin-induced lung fibrosis.

\section{RESULTS}

Mesenchymal cells increase periostin mRNA expression after bleomycin treatment

To specifically assess the upregulation of periostin expression in lung mesenchymal cells after bleomycin treatment, WT mice were given intratracheal saline or bleomycin on day 0 . All lungs were harvested on day 14 and mesenchymal cells were cultured for 14 days then sorted into fibrocytes (CD45 positive) and fibroblasts (CD45 negative). RNA was isolated for real-time RT-PCR analysis. We observed that both fibroblasts and fibrocytes had significant increases in periostin mRNA expression post bleomycin treatment (Figure 1).

\section{TGF $\beta 1$ and periostin co-regulate each other in lung mesenchymal cells}

We previously showed that treatment of WT mesenchymal cells with TGF $\beta 1$ showed a significant increase in periostin production. ${ }^{33}$ To assess whether periostin also up-regulates TGF $\beta 1$ production in purified fibroblasts and fibrocytes, we isolated these cell types from lung mince cultures from naïve mouse lungs and treated with recombinant periostin $(500 \mathrm{ng} / \mathrm{mL})$ in SFM. Treatment of WT fibrocytes and fibroblasts with periostin lead to increased TGF $\beta 1$ production as measured by ELISA (Figure 2a). In addition, treatment of fibroblasts and fibrocytes with recombinant TGF- $\beta 1(2 \mathrm{ng} / \mathrm{ml})$ increased protein production of periostin as measured by ELISA (Figure 2b), as well as increased mRNA expression of periostin (Figures $\mathbf{2 c}$ and $\mathbf{d}$ ).

Since, periostin and TGF $\beta 1$ seem to be co-regulating each other, we wanted to assess whether the production of collagen I from fibroblasts and fibrocytes was different in the presence of periostin and if this effect was dependent on TGF- $\beta 1$ signaling. Figure 3 illustrates that treatment of murine lung mesenchymal cells with periostin for $48 \mathrm{~h}$ led to a significant increase in mRNA expression for collagen 1 . Because collagen 1 mRNA is extremely stable, ${ }^{39}$ small increases in transcription can lead to significant enhancement of translated protein. However, the 
a
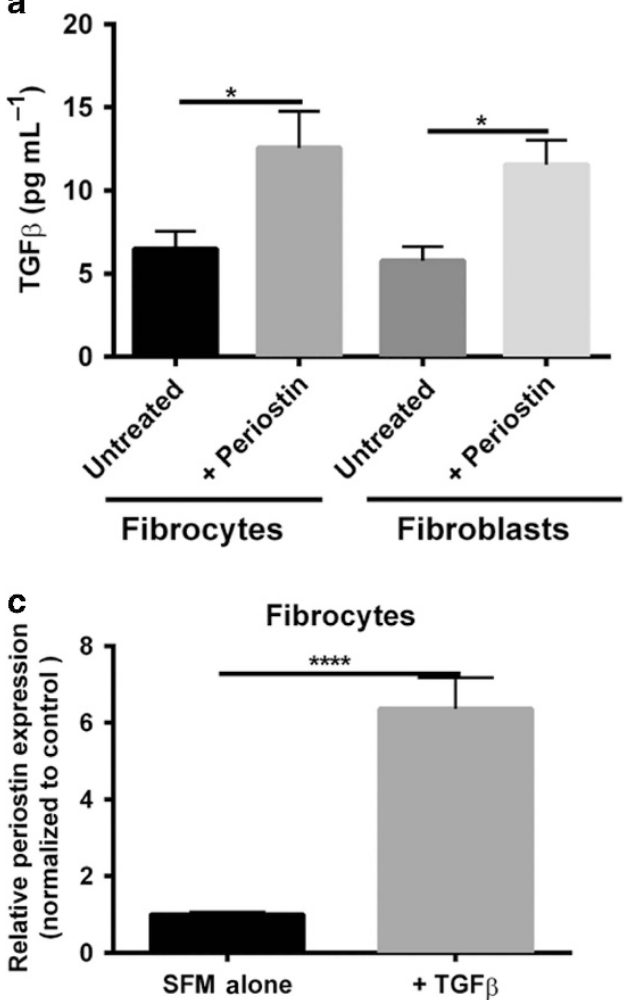

b

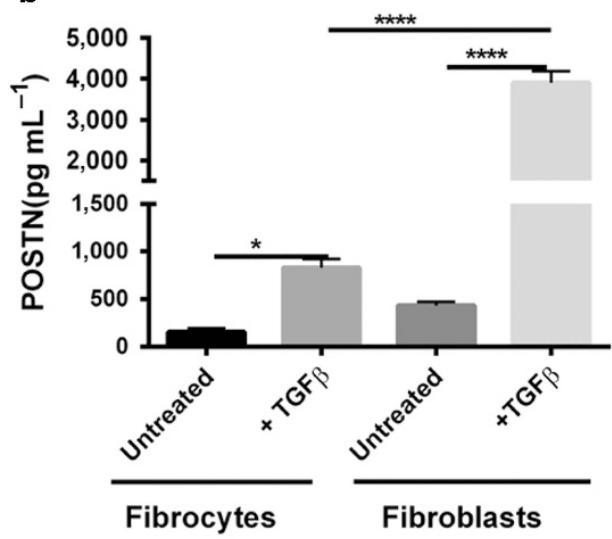

d

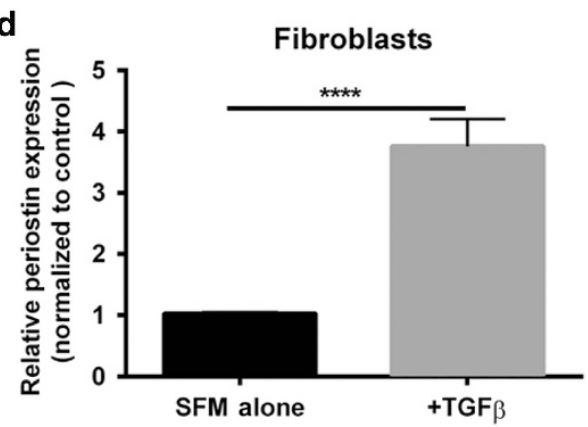

Figure 2 Periostin and TGF $\beta$ regulate each other in fibroblasts and fibrocytes. Fibroblasts and fibrocytes from wild-type mice were treated with periostin $(500 \mathrm{ng} / \mathrm{ml})$ or TGF $\beta(2 \mathrm{ng} / \mathrm{ml})$ for $48 \mathrm{~h}$. Cell-free supernatants were collected and analyzed by ELISA for TGF $\beta$ (a) and periostin (b). Total RNA was isolated and by real time RT-PCR we measured the expression of periostin and $\beta$-actin in fibrocytes (c) and fibroblasts (d). Data are representative of mean \pm s.e.m., $n=3$ wells/treatment per group. ${ }^{\star} P<0.05,{ }^{\star \star} P<0.01,{ }^{* \star \star \star} P<0.0001$, ns $=$ not significant.

increase in collagen 1 expression was independent of TGF $\beta 1$ signaling in the fibrocytes compared to the fibroblasts as indicated by treatment with the A8301 TGF- $\beta 1$ receptor antagonist. Additionally, Supplementary Figure 1 online demonstrates that periostin can influence other extracellular matrix components as well including collagen 3 and fibronectin. Overall these data suggest that TGF $\beta 1$ and periostin are co-regulating each other but periostin can signal to fibrocytes independently of TGF $\beta 1$ and that the receptor needed for periostin signaling may be different on fibrocytes compared to fibroblasts.

\section{Decreased integrin expression in fibrocytes from periostin $^{-l-}$ mice post-bleomycin treatment}

In many cancers, periostin binds to integrins activating the $\mathrm{AKT} / \mathrm{PKB}$ and FAK-mediated signaling pathways. This leads to increased cell survival, invasion, angiogenesis, metastasis and epithelial-mesenchymal transition. ${ }^{40}$ Periostin also functions as a ligand for integrin alphaVbeta3 and alphaVbeta 5 to support adhesion and migration of epithelial cells. ${ }^{41}$ Because our data suggest that periostin might be signaling differently in fibrocytes vs fibroblasts, we wanted to examine the expression levels of integrins in WT and periostin ${ }^{-/-}$naïve lung mesenchymal cells. As illustrated in Figures $\mathbf{4 a - d}$, there is no significant difference in the baseline mRNA expression levels of alpha 1 , alpha $\mathrm{V}$, beta 1 and beta 5 integrin levels on fibrocytes and fibroblasts from WT or periostin ${ }^{-/-}$mice, although in most cases, the expression levels of integrins were lower in fibrocytes than fibroblasts. We next treated WT and periostin $^{-1-}$ mice intratracheally with saline or bleomycin for 14 days then cultured lung mesenchymal cells, sorted for fibrocytes and assessed mRNA expression for different integrins post-bleomycin treatment. Alpha 1, alpha V and beta 1 were found to be significantly upregulated in WT fibrocytes post-bleomycin treatment but beta 5 expression was not altered. None of the integrins were upregulated post-bleomycin in the periostin ${ }^{-1-}$ cells (Figures $4 \mathbf{e}-\mathbf{h}$ ). To compare the differences in integrin expression on fibrocytes at the protein level, fibrocytes were isolated from bleomycin-or saline-treated WT or periostin ${ }^{-1}$ mice and integrin profiles were analyzed by flow cytometry (Figures 5a-c). Levels of alpha 1 were lower in periostin ${ }^{-/-}$fibrocytes but did not reach significance in this experiment. In contrast, the decrease in $\beta 1$ integrin noted in periostin ${ }^{-1-}$ mice did show a significant reduction (Figure 5c). We also confirmed lower levels of $\beta 1$ integrin in periostin ${ }^{-1-}$ fibrocytes by Western blot (Figure 5d). There were no statistically significant differences in integrin expression in fibroblasts from WT or periostin $^{-/}$mice at the mRNA or protein levels (Supplementary Figure 2). To determine how integrin expression 
a

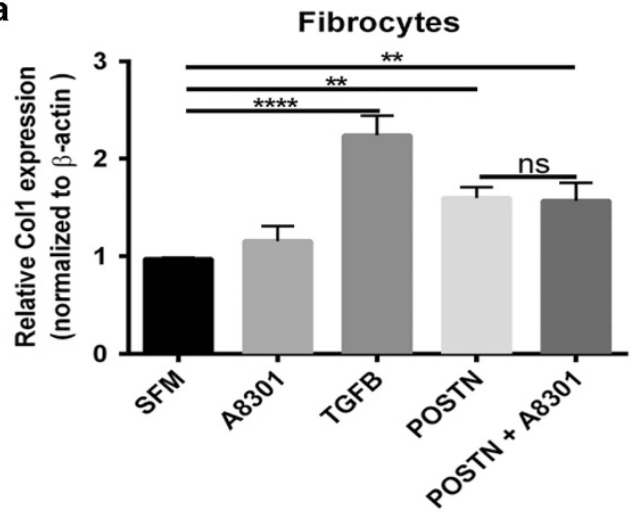

b

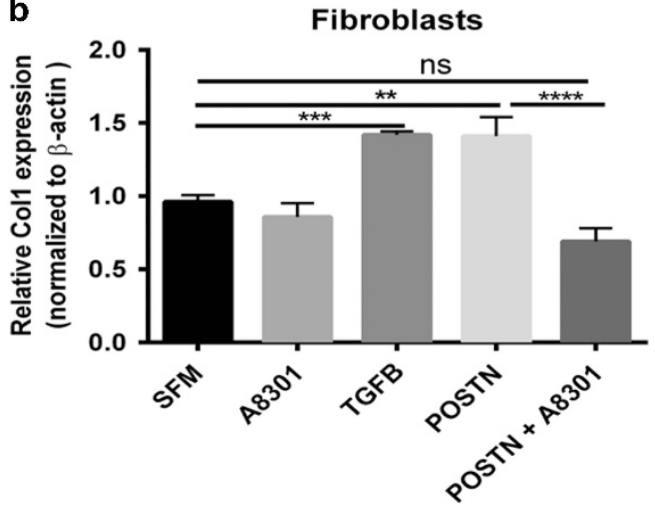

Figure 3 Periostin induces collagen 1 expression in fibrocytes independently of TGF $\beta$ signaling. Mesenchymal cells were cultured in complete media, sorted into fibrocytes and fibroblasts, then switched to SFM where they were treated with TGF $\beta(2 \mathrm{ng} / \mathrm{ml})$, A8301 (ALK5 inhibitor) or periostin $(500 \mathrm{ng} / \mathrm{ml})$ for $48 \mathrm{~h}$. Total RNA was isolated and by real time RT-PCR we measured the expression of collagen 1 and $\beta$-actin in fibrocytes (a) and fibroblasts (b). Data are representative of mean \pm s.e.m., $n=3$ wells/treatment per group from 2 independent experiments ${ }^{* * * *} P<0.0001,{ }^{* * *} P<0.001,{ }^{* *} P<0.01,{ }^{*} P<0.05$ and $\mathrm{ns}=$ not significant.

profiles correlated with TGF $\beta 1$ production, we treated fibrocytes with exogenous TGF $\beta 1$ and found increased mRNA expression for alpha V, alpha 1 and beta 5 in wildtype cells (Figure 6). Expression of these integrins was similarly increased in the periostin $^{-1-}$ cells treated with TGF $\beta 1$. In contrast, TGF $\beta 1$ treatment caused a modest, but not significant increase in beta 1 expression in WT cells, but did not in periostin $^{-1-}$ cells providing further support that beta 1 integrin expression may be necessary for periostin signaling to fibrocytes in murine lungs (Figure 6). Next, we treated WT fibrocytes and fibroblasts with periostin in the presence of a beta 1 integrin blocking antibody (102209-Biolegend) and after $48 \mathrm{~h}$ assessed collagen 1 mRNA expression as an indication of mesenchymal cell activation (Figure 7). Fibrocytes treated with periostin in the presence of beta 1 blocking antibody had markedly less collagen 1 mRNA expression compared to samples treated with periostin and isotype control (Figure 7a). In contrast, addition of beta 1 integrin blocking antibody to fibroblasts showed no change in collagen 1 expression (Figure $7 \mathbf{b}$ ). Taken together our data suggest periostin signals collagen expression through beta 1 integrin to activate fibrocytes and this is independent of TGF $\beta$ signaling.

Fibrocytes exacerbate bleomycin-induced fibrosis through paracrine effects and in a periostin-dependent manner

We previously showed that fibrocytes augment FITC-induced lung fibrosis. ${ }^{24}$ In addition, fibrocytes and monocytes were the major producers of periostin in circulation and a hematopoietic source of periostin was important for fibrogenesis in the bleomycin model of lung fibrosis. ${ }^{33}$ To specifically assess the role of periostin produced by fibrocytes in lung fibrogenesis, $\mathrm{WT}_{\text {or periostin }}{ }^{-1-}$ mice were given intratracheal saline or bleomycin on day 0 . On day 5 post-bleomycin, mice were given $5 \times 10^{5}$ additional cultured WT or periostin ${ }^{-/-}$lung fibrocytes. Lungs from all mice were harvested on day 21 post-bleomycin and lung collagen content was measured by hydroxyproline assay. As expected, bleomycin-treated WT mice showed a significant increase in collagen content when compared to saline controls but WT mice that received the additional WT fibrocytes had significantly higher amounts of collagen in the lungs compared to bleomycin alone (Figure 8a). Interestingly, bleomycin-treated WT mice given the periostin ${ }^{-1-}$ fibrocytes showed similar levels of lung collagen as the bleomycin-treated wild-type mice with no added cells (Figure $\mathbf{8 a}$ ). Figure $\mathbf{8 b}$ demonstrates that periostin $^{-/-}$mice treated with bleomycin then given additional WT lung fibrocytes have significantly more collagen content in the lungs compared to periostin ${ }^{-1-}$ mice treated with saline or bleomycin alone. Additionally, we analyzed the ability of fibrocytes to maintain their CD45 expression post-transfer over time. Using flow cytometry analysis we found that all the labeled $\mathrm{PKH} 26$ positive fibrocytes were detected as CD45 positive cells 4-8 days after transfer (Figure 8c). These data demonstrate that fibrocytes were not differentiating into CD45-negative fibroblasts after transfer.

\section{Periostin production by fibrocytes promotes their} profibrotic effects on myofibroblasts via other mediators Myofibroblast differentiation is characterized, in part, by induction of $\alpha \mathrm{SMA}$ gene expression as well as by increased production of ECM components and fibrogenic cytokines such as TGF $\beta 1 .^{40-42}$ To assess how fibrocyte-derived periostin affects myofibroblast differentiation, we analyzed protein expression of $\alpha \mathrm{SMA}$ in WT untreated fibroblasts that were incubated with cell-free supernatants from CD45 positive fibrocytes from bleomycin-treated WT and periostin ${ }^{-1}$ mice. Cells were incubated in this media for $24 \mathrm{~h}$. After $24 \mathrm{~h}$ we analyzed expression of $\alpha$ SMA by Western blot and saw less aSMA expression in WT fibroblasts that were incubated with bleomycin-treated supernatants from periostin ${ }^{-1-}$ fibrocytes (Figure 9a). To address whether this effect was solely due to periostin or possibly involved other downstream signaling proteins we performed a microarray using a targeted mouse fibrosis array with the goal of identifying differences between the WT and periostin ${ }^{-1-}$ fibrocytes. We saw significant decreases in connective tissue growth factor (CTGF) and Lysyl oxidase (LOX) but not platelet-derived growth factor (PDGF) $\alpha$ 

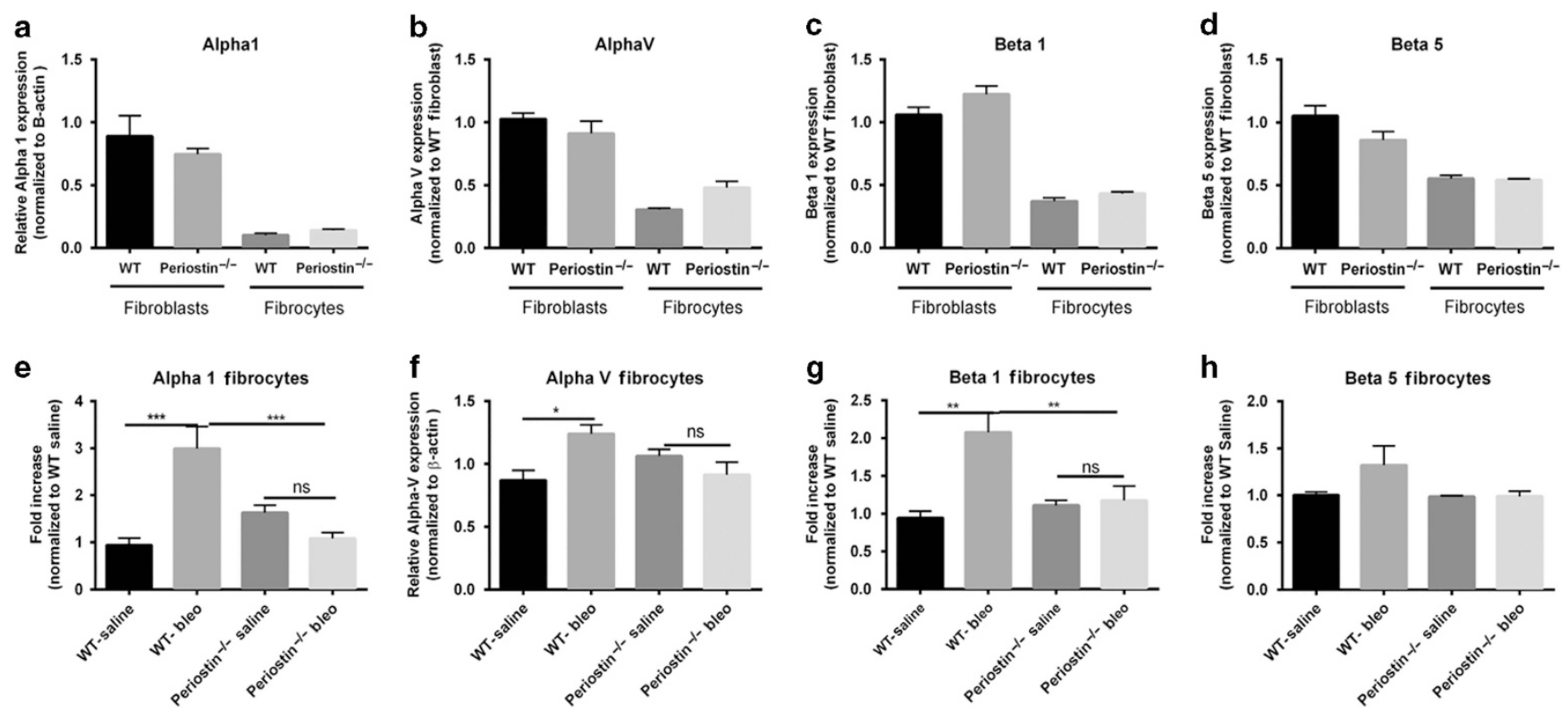

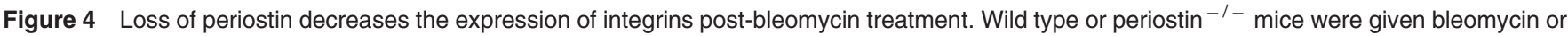
saline intratracheally on day 0 . On day 14 lungs were harvested and mesenchymal cells were cultured and sorted as previously described. Total RNA was isolated and by real time RT-PCR we measured the expression of alpha1, alpha V, beta 1 and beta 5 expression in saline treated fibrocytes and fibroblasts (a-d) and bleomycin treated (e-h) fibrocytes. Values are expressed as mean \pm s.e.m., and represent $n=3$ animals/group from two independent experiments $\mathrm{ns}=$ not significant, ${ }^{* \star} P<0.001,{ }^{* *} P<0.01$ and ${ }^{*} P<0.05$.
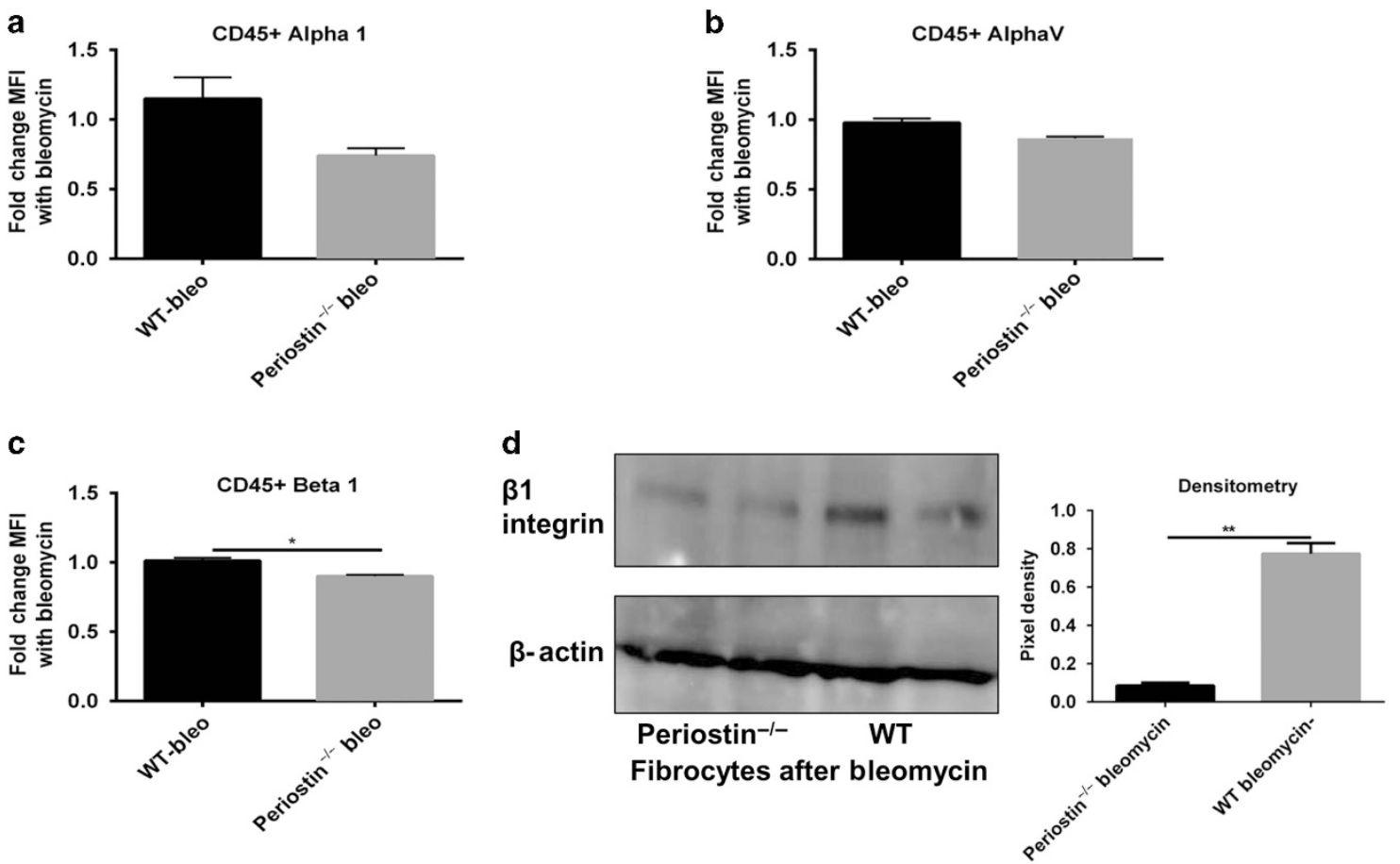

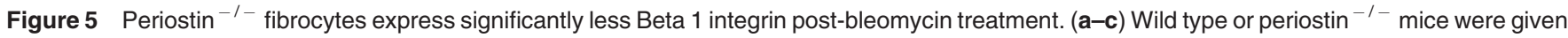
bleomycin or saline intratracheally on day 0 . On day 14 lungs were harvested and mesenchymal cells were incubated with a pan CD45 antibody, alpha 1 , alpha $V$ and beta 1-integrin antibodies. Cells were analyzed by flow cytometry to assess surface expression of integrins on CD45 + cells. Graphs show the expression of integrins in bleomycin-treated samples relative to the saline-treated samples of each genotype. (d) Fibrocytes from bleomycin-treated WT and periostin ${ }^{-1-}$ mice were lysed in RIPA buffer with protease inhibitor and we assessed the expression of Beta 1 and $\beta$-actin by Western blot The intensity of the bands were also quantitated using the $\mathrm{NIH}$ Image $\mathrm{J}$ software from duplicate cultures, ${ }^{\star *} P<0.01$ and ${ }^{\star} P<0.05$. 

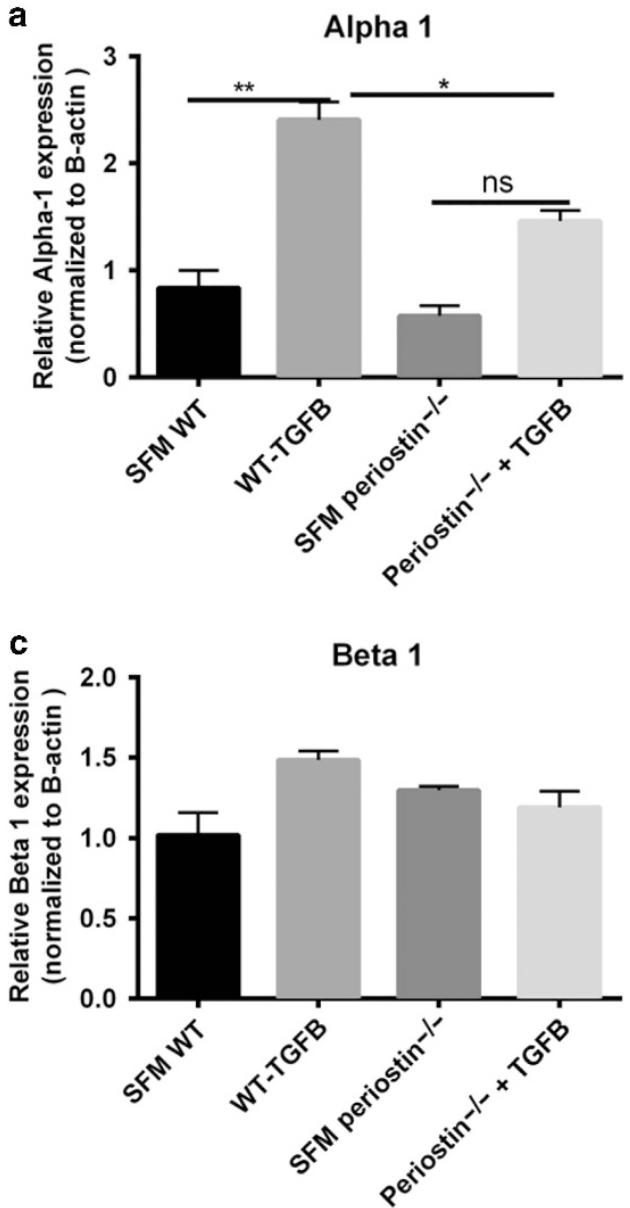
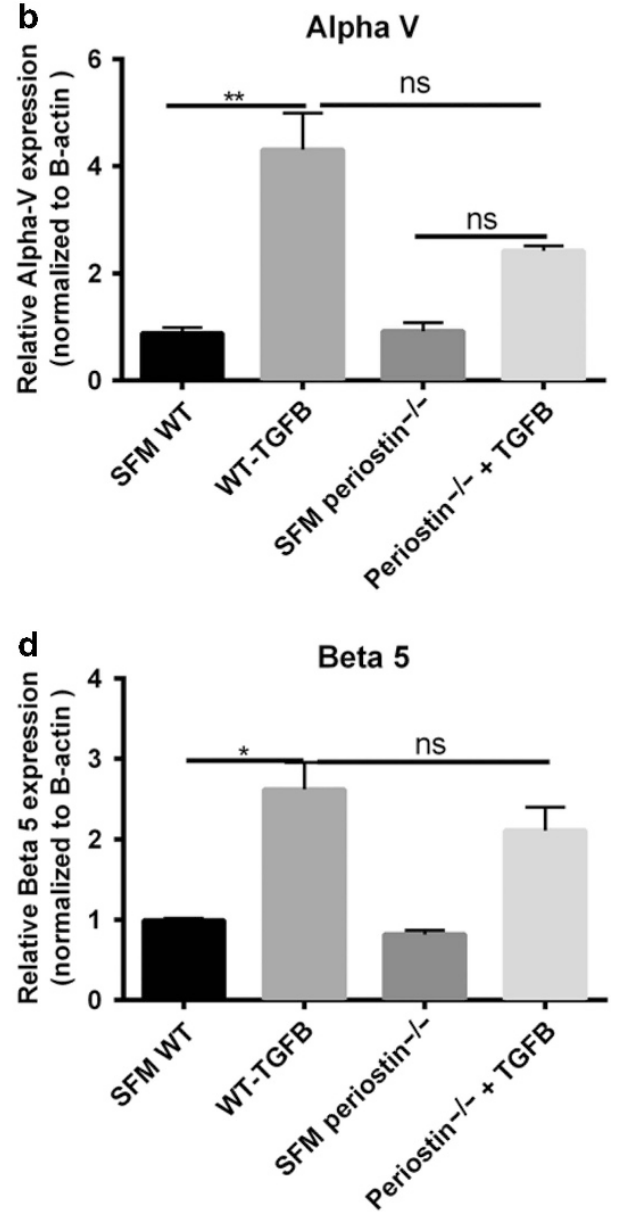

Figure 6 TGF $\beta$ treatment did not induce $\beta 1$ integrin mRNA expression in fibrocytes in the absence of periostin. Fibrocytes from wild-type and periostin $^{-1-}$ mice were treated with recombinant TGF $\beta 1(2 \mathrm{ng} / \mathrm{ml})$ for $48 \mathrm{~h}$. Total RNA was isolated and by real time RT-PCR we measured the expression of different integrins and $\beta$-actin in fibrocytes (a) Alpha 1, (b) Alpha V, (c) Beta 1 and (d) Beta 5. Data are representative of mean \pm s.e.m., $n=3$ wells/treatment per group, ${ }^{* *} P<0.01,{ }^{*} P<0.05$ and ns $=$ not significant.

in periostin $^{-1-}$ fibrocytes. Findings were confirmed by RT-qPCR (Figure 9b). In addition, there was a significant increase in CTGF mRNA expression in fibrocytes treated with exogenous periostin; however, in the presence of a beta 1 blocking antibody, periostin could no longer induce a significant increase in CTGF (Figure 9c). There was no change in CTGF mRNA expression in fibroblasts (Figure 9d). Given the importance of CTGF in fibrogenesis, we measured the amount of CTGF protein in the supernatants used in the experiments described in Figure 9a by ELISA and saw less CTGF in the periostin ${ }^{-1-}$ fibrocytes supernatants (Figure 9e). Taken together these data suggest that fibrocytes in the presence of periostin secrete additional pro-fibrotic mediators such as CTGF that contribute to myofibroblast differentiation.

\section{Periostin signaling is critical for fibrocyte amplification of a profibrotic response}

We next wanted to determine the impact of periostin signaling to the fibrocytes to induce their profibrotic paracrine mediators. We isolated fibrocytes from bleomycin-treated wild-type mice and cultured them in SFM overnight in the presence or absence of an anti-periostin $\mathrm{Ab}$ to neutralize the periostin that is secreted into the supernatant by fibrocytes. When these supernatants were collected and incubated with fibroblasts, the supernatant collected from fibrocytes treated with anti-periostin antibodies could not induce expression of $\alpha$-SMA to the same extent in the fibroblasts as supernatant from untreated fibrocytes (Figure 10). These results suggest that autocrine periostin signaling to the fibrocytes themselves is a critical upstream mediator of the release of fibrocyte pro-fibrotic paracrine mediators.

\section{DISCUSSION}

Previous studies have shown that periostin ${ }^{-1-}$ mice treated with bleomycin intratracheally had decreased collagen content compared with WT littermates. ${ }^{33,43}$ Both monocytes and fibrocytes likely contribute to the pool of circulating periostin in IPF patients and chimeric mouse studies demonstrated that both the hematopoietic-derived and structural sources of periostin contributed similarly to fibrogenesis. ${ }^{33}$ These studies suggested that a circulating source of periostin could be playing a significant role in the exacerbation of lung fibrosis, but it was 
a

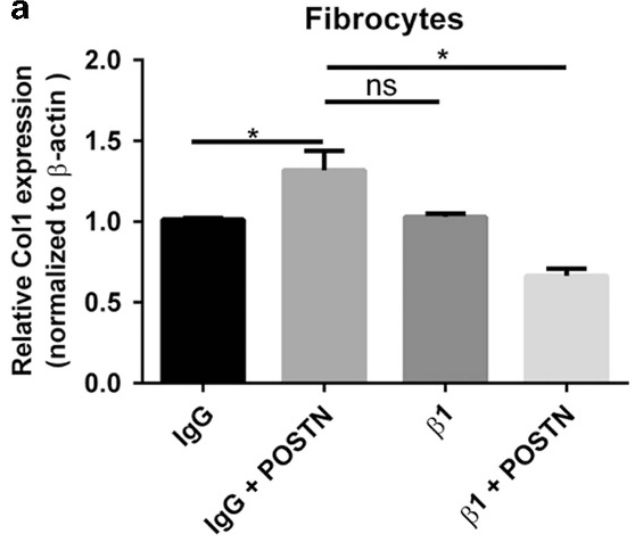

b

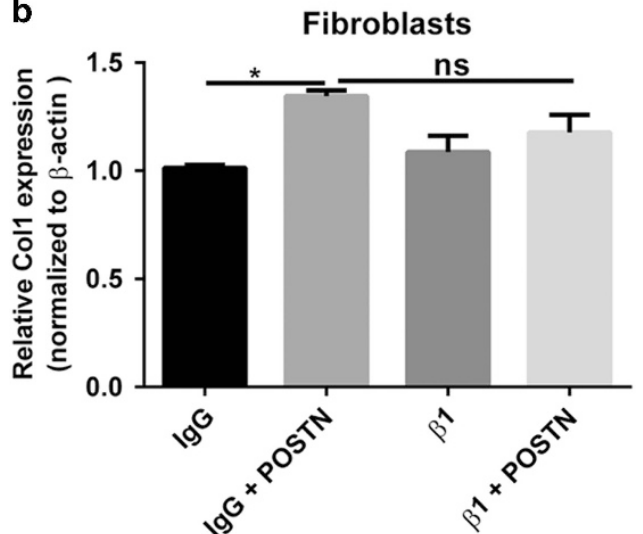

Figure 7 Beta 1 integrin blockade in WT fibrocytes caused decreased Collagen 1 expression with periostin treatment but showed no effect in fibroblasts. Mesenchymal cells were cultured in complete media, sorted into fibrocytes and fibroblasts, then switched to SFM where they were treated with purified rat anti-mouse beta 1 ( $\beta 1)(H M \beta 1-1$, rat CD29) blocking antibody or purified armenian Hamster IgG (400916) isotype control for 30 mins, then incubated with or without periostin $(500 \mathrm{ng} / \mathrm{ml})$ for $48 \mathrm{~h}$. Total RNA was isolated and by real time RT-PCR we measured the expression of collagen 1 and $\beta$-actin in fibrocytes (a) and fibroblasts (b). Data are representative of mean \pm s.e.m., $n=3$ wells/treatment per group ${ }^{*} P<0.05$ and $n s=$ not significant

unclear how the lower levels of periostin produced by circulating fibrocytes vs. structural cells (e.g. fibroblasts, see Figure 2b) were influencing disease. Our current findings in vivo, and in vitro, suggest that fibrocyte profibrotic function is likely via multiple paracrine mechanisms.

We examined the effects of fibrocytes in the exacerbation of bleomycin-induced fibrosis in WT and periostin ${ }^{-1-}$ mice. Adoptive transfer of WT fibrocytes during established lung fibrosis augmented bleomycin-induced fibrosis in recipient mice, corroborating the earlier studies in a fluorescein isothiocyanate (FITC)-induced model of pulmonary fibrosis. ${ }^{24}$ Furthermore, in periostin ${ }^{-1-}$ mice receiving WT fibrocytes there was a significant increase in lung hydroxyproline, which is evidence of enhanced lung fibrosis relative to the periostin ${ }^{-1-}$ mice that did not receive additional fibrocytes (Figure 8). We first wondered whether there was a defect in migration of periostin $^{-1-}$ fibrocytes to the injured lung. However, Figure 8c shows that periostin ${ }^{-1-}$ fibrocytes could be identified in the lungs as easily as WT fibrocytes. Furthermore, we found no defect in the expression of chemokine receptors on the WT vs. the periostin $^{-1-}$ fibrocytes (data not shown). Therefore, the decrease in fibrosis in mice treated with the periostin ${ }^{-/-}$fibrocytes could not be attributed to a migration defect. Furthermore, we considered the possibility that WT fibrocytes might better be able to differentiate into fibroblasts or myofibroblasts once arriving in the injured lung.

While in vitro work has clearly shown that fibrocytes can differentiate into myofibroblasts, ${ }^{25,44,45}$ our in vivo work corroborates recent findings in another model system to suggest fibrocytes are not differentiating into myofibroblasts during fibrosis (Figure 8c). ${ }^{32}$ We also previously showed that fibrocytes migrate to areas in the lungs adjacent to fibroblasts. ${ }^{23}$ Given that collagen production by circulating cells is also dispensable for development of lung fibrosis, ${ }^{28}$ these data, when taken together, suggest that fibrocytes work mostly by paracrine mechanisms to induce fibrogenesis. We believe that their ability to migrate to the site of injury by virtue of their chemokine receptors makes them uniquely poised to deliver their pro-fibrotic paracrine factors where they are most effective at stimulating wound repair; yet in the setting of chronic lung disease, these fibrocytes may promote exaggerated wound repair and fibrosis.

It is likely that some of the paracrine effects of periostinproducing fibrocytes are due to periostin itself. We have previously shown that periostin can directly influence ECM deposition and migration of lung fibroblasts ${ }^{33}$ (and Supplementary Figure 1). Another feature of myofibroblasts is their ability to resist apoptosis, likely due to upregulation of antiapoptotic proteins such as X-linked inhibitor of apoptosis (XIAP) expression. ${ }^{46}$ Periostin may directly contribute to myofibroblast resistance to apoptosis via stimulating XIAP protein expression (Supplementary Figure 3). We recently demonstrated that blocking inhibitor of apoptosis family proteins using pharmacologic interventions attenuated bleomycin-induced fibrosis, ${ }^{46,47}$ Thus, periostin likely contributes to the exacerbation of bleomycin-induced fibrosis at least in part via direct effects on fibroblasts.

Another possibility is that periostin signals back to the fibrocytes themselves to influence production of other profibrotic mediators. Figure $\mathbf{1 0}$ demonstrates that secretion of pro-fibrotic mediators by fibrocytes is blunted in the presence of a neutralizing antibody against periostin. This suggests that periostin plays an important autocrine signaling role to fibrocytes to promote pro-fibrotic mediator release. Additionally, we demonstrate that periostin production by fibrocytes promotes expression of CTGF and LOX post bleomycin-treatment (Figure 9b) and fibrocytes secrete less CTGF in the absence of periostin post-bleomycin treatment (Figure 9e). CTGF has previously been described as an activator of mesenchymal cells, ${ }^{48-50}$ while LOX is known to promote collagen crosslinking, likely leading to stiffer ECM and more robust matrix-dependent fibroblast activation. ${ }^{34,51}$ CTGF can induce a variety of cytokines such as TGF $\beta$ and vascular endothelial growth factor (reviewed in ref. 48) which in turn 

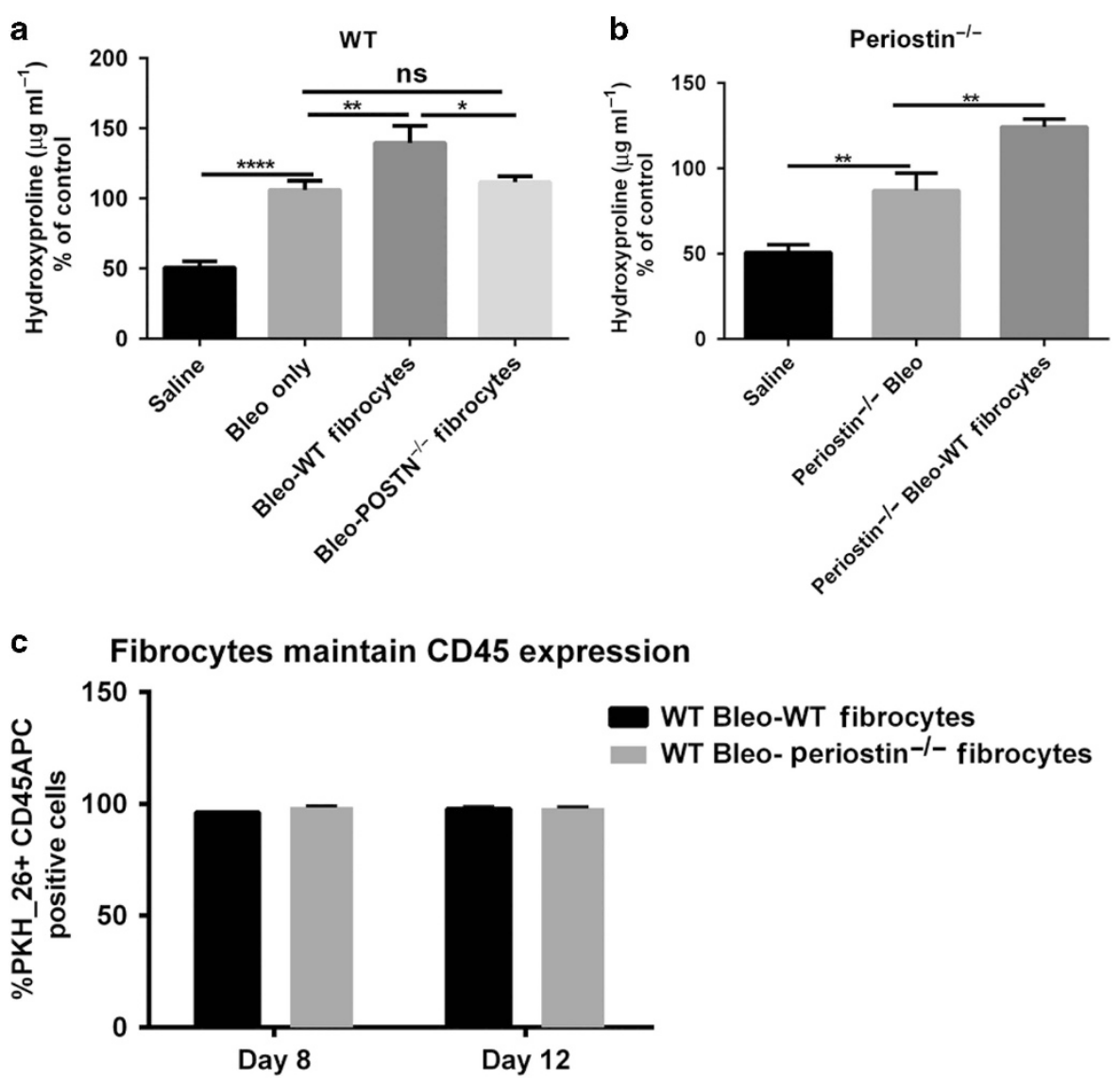

Figure 8 Adoptive transfer of WT but not periostin ${ }^{-1-}$ fibrocytes augments bleomycin-induced fibrosis but both maintained CD45 expression in vivo. (a) Wild type mice were given $0.025 \mathrm{U}$ of bleomycin or PBS intratracheally on day 0 . On day 5 , post-bleomycin treatment half of each group received $5 \times 10^{5}$ WT or periostin ${ }^{-1}$ - fibrocytes by intravenous tail vein injection. Lungs were harvested on day 21 post-bleomycin for hydroxyproline quantification. Data shown are pooled from two independent experiments, with $n=4-6$ mice per group in each experiment. ${ }^{*} P<0.01$, ${ }^{*} P<0.05$ and ns $=$ not significant (b) Periostin ${ }^{-1-}$ mice were given $0.025 \mathrm{U}$ of bleomycin intratracheally on day 0 . On day 5 post-bleomycin treatment, half of each group received $5 \times 10^{5} \mathrm{WT}$ fibrocytes, lungs were harvested and assessed for collagen content on day 21 by hydroxyproline assay ${ }^{*} P<0.05$. (c) Wild type mice were given $0.025 \mathrm{U}$ of bleomycin or PBS intratracheally on day 0 . On day 5, post-bleomycin treatment half of each group received $5 \times 10^{5} \mathrm{PKH}-26$ labeled WT or periostin ${ }^{-1-}$ fibrocytes by intravenous tail vein injection. On day 3 and 7 post-transfer, lungs were then harvested and total lung leukocytes were enumerated and then labeled with CD45 APC to determine the percentages of PKH-26 + CD45 + APC fibrocytes. Data shown represents mean \pm s.e.m., $n=3$ animals/group, ns, not significant.

induce more expression of CTGF, indicating a positive feedback loop involving CTGF expression that can contribute to the progression of fibrosis. It is also likely that a similar feedback loop is occurring with periostin. Inhibiting this positive feedback loop would be ideal for understanding the mechanism by which CTGF from fibrocytes enables persistent lung fibrosis and promotes myofibroblast differentiation in the presence of periostin. However, there is not a specific receptor antagonist for CTGF due to the complex mechanism of CTGF signaling. Treatment of duplicate cultures of WT untreated fibroblasts with $10 \mu \mathrm{M}$ of an MRTF inhibitor (CCG 203971, shown to inhibit CTGF expression), in the presence of WT bleomycin fibrocyte supernatant resulted in a decrease in $\alpha$ SMA protein expression similar to what was seen with the periostin ${ }^{-1-}$ supernatants demonstrating the importance of autocrine CTGF expression in fibroblasts (Supplementary Figure 4).

To further examine the effects of periostin on fibrocytes we designed experiments using integrin blocking antibodies. Our results suggest that periostin signals uniquely to fibrocytes via beta 1 integrin. A recent study by Reed and colleagues ${ }^{52}$ suggested that inhibition of the alphaVbetal integrin using a small molecule inhibitor significantly attenuated bleomycininduced pulmonary fibrosis. In that study the authors only looked at lung mesenchymal cells, which are a combination of fibroblasts and fibrocytes. Given our findings that beta1 integrins are critical for periostin signaling to fibrocytes, it will be important to determine if the alphav-beta 1 heterodimers are responsible for periostin signaling to fibrocytes.

One very interesting experiment would have been to deplete fibrocytes during fibrosis; however this strategy proved technically unsuccessful. We generated a collagen $1 \alpha 2$-CreDTR transgenic mouse and used these animals to generate bone-marrow chimeras in a CD45.2 recipient mouse so that the bone-marrow derived cells (but not lung resident cells) would be expressing CD45.1 and the collagen $1 \alpha 2$-Cre-DTR transgene. These mice were then given bleomycin and treated with diphtheria toxin on days 11 and 18 to deplete the recruited collagen 1 $\alpha 2$-Cre-DTR expressing cells. Despite excellent 
a

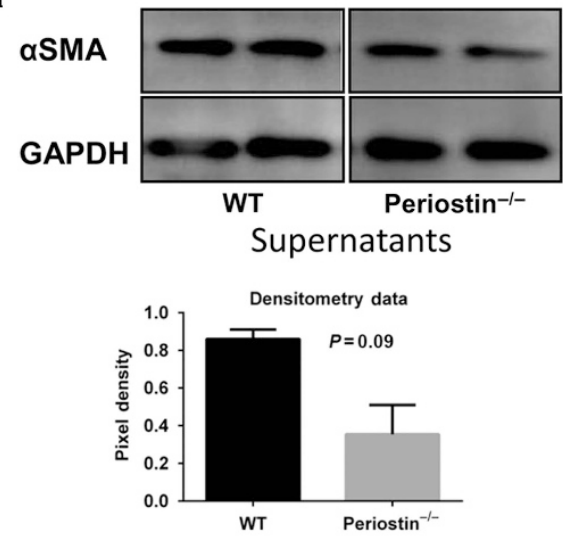

b
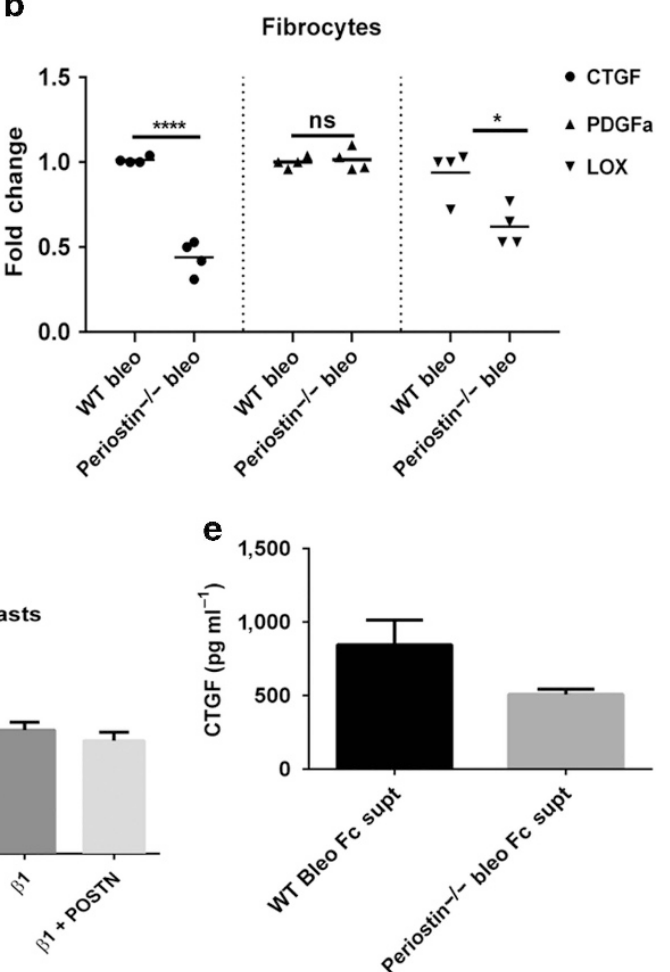

Figure 9 WT fibrocytes secrete CTGF in the presence of periostin and increase $\alpha$ SMA protein expression in fibroblasts in the presence of periostin. (a) Wild type mice were given $0.025 \mathrm{U}$ of bleomycin or PBS intratracheally on day 0 . On day 14, post-bleomycin treatment lung mesenchymal cells were cultured for 14 days. After 14 days in culture cells were sorted and CD45 positive fibrocytes were incubated in SFM overnight. Cell-free supernatants were collected from both bleomycin-WT and periostin ${ }^{-1-}$ cells and added 1:1 with SFM onto WT untreated fibroblasts (CD45 negative) cells for $24 \mathrm{~h}$. Cells were lysed in RIPA buffer with protease inhibitor and we assessed the expression of $\alpha$ SMA and GAPDH by Western blot. Densitometry data is also shown. (b) Total RNA was isolated from the WT and periostin ${ }^{-1-}$ fibrocytes after overnight incubation in serum-free media and by real time RT-PCR we measured the mRNA expression of CTGF, PDGF $\alpha$ and LOX. Data shown represents mean \pm s.e.m., $n=3$ wells $/$ group, ns $=$ not significant, ${ }^{* * * \star} P<0.0001,{ }^{* \star} P<0.01$ and ${ }^{*} P<0.05$. (c-d) Total RNA was isolated from WT mesenchymal cells treated with periostin $(500 \mathrm{ng} / \mathrm{ml})$ and by real time RT-PCR we measured the mRNA expression of CTGF. Data shown represents mean \pm s.e.m., $n=3$ wells/group, ns $=$ not significant and ${ }^{*} P<0.05$. (e) Cell free supernatants from fibrocytes $(\mathrm{Fc})$ were analyzed by ELISA for CTGF.

chimerism in the lung on day 21 , surprisingly, there was a high percentage of recipient fibrocytes present post-bleomycin treatment that could not be eliminated by diphtheria toxin treatment (Supplementary Figure 5). Additionally, the low level of DTR expression driven by the collagen promoter in fibrocytes likely impaired the ability to deplete these cells with diphtheria toxin. We feel it is important for the lung community to know that this strategy to create a fibrocyteknock-out mouse was not effective. Definitive proof of the role of fibrocytes in lung fibrosis awaits the characterization of a fibrocyte-specific promoter that can be used in future cellular depletion studies.

In conclusion, the current study showed that once recruited to the lungs, fibrocytes did not differentiate into fibroblasts directly. Rather, fibrocyte-derived periostin signals via beta 1 integrin to cause release of profibrotic mediators such as CTGF from the fibrocytes themselves to promote myofibroblast differentiation.

\section{METHODS}

Animals. Wild-type C57BL/6 (B6) age and sex-matched mice were obtained from The Jackson Laboratory (Bar Harbor, ME).
Periostin $^{-1-}$ mice were bred at the University of Michigan. In previous experiments we verified that $\mathrm{C} 57 \mathrm{Bl} / 6$ mice purchased from Jackson Laboratory behave similarly with respect to the periostin $^{-1-}$ mice as do control mice generated by heterozygous breeding in house. Mice were housed under pathogen-free conditions and provided food and water ad libitum. All animal experiments complied with university and federal guidelines for humane use and care. The University of Michigan Institutional Animal Care and Use Committee approved these experiments.

Bleomycin model of pulmonary fibrosis. Mice were given bleomycin ( $0.025 \mathrm{U}$ (Sigma) dissolved in sterile saline in a $50-\mu \mathrm{l}$ volume) intratracheally as described previously ${ }^{53}$ or via oropharyngeal aspiration.

Lung collagen measurements. Collagen deposition was measured using a hydroxyproline assay on day 21 post-bleomycin challenge as described previously. ${ }^{53}$

Semi quantitative real-time RT-PCR. RNA was extracted from lung mesenchymal cells using TRIzol reagent (Invitrogen, Carlsbad, CA) then analyzed by real-time RT-PCR on an Applied Biosystems StepOne Plus thermocycler (Applied Biosystems, Foster City, CA). Gene specific primers and probes were purchased from Sigma-Aldrich (St Louis, MO). Relative expression was calculated using the comparative $C_{T}$ method as previously described. ${ }^{54}$ Additionally, RNA isolated from bleomycin-treated WT and periostin ${ }^{-1-}$ fibrocytes was 


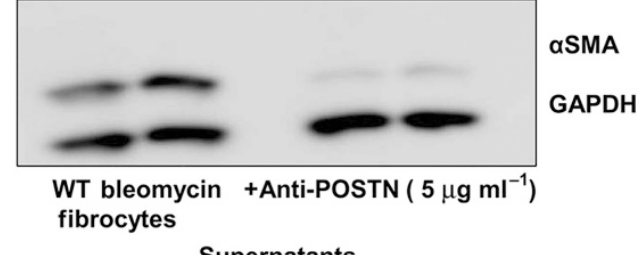

Supernatants

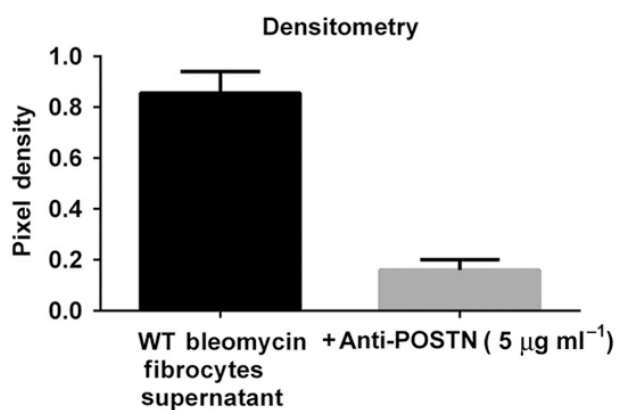

Figure 10 Neutralization of periostin secretion by fibrocytes postbleomycin treatment limited their ability to promote myofibrolast differentiation. WT mice were given $0.025 \mathrm{U}$ of bleomycin or PBS intratracheally on day 0 . On day 14 , post-bleomycin treatment lung mesenchymal cells were cultured for 14 days. After 14 days in culture cells were sorted and CD45 positive fibrocytes were incubated in SFM overnight in presence or absence of a mouse periostin neutralizing antibody (AF-2955, R\&D systems). Cell-free supernatants were collected from both and added 1:1 with SFM onto WT untreated fibroblasts (CD45 negative) for $24 \mathrm{~h}$. Cells were lysed in RIPA buffer with protease inhibitor and we assessed the expression of $\alpha$ SMA and GAPDH by western blot. Data was quantitated using NIH Image $\mathrm{J}$ software and pixel density is represented on bar graph.

analyzed using the Mouse Fibrosis $\mathrm{RT}^{2}$ Profiler PCR Array and then confirmed using gene specific primers and probes (see on line Supplementary Table 1) as previously described. We have determined that $\beta$-actin expression did not change significantly in response to TGF $\beta$ stimulation (data not shown) making this an appropriate normalization for our gene expression data.

Enzyme-linked Immunoassay/ELISA. Whole lung homogenates were prepared for analysis of cytokines as described previously. ${ }^{53}$ Each ELISA assay was done using a Duoset ELISA kit (R\&D systems, Minneapolis, MN) according to manufacturer's instructions.

Mesenchymal cell isolation. Lung mesenchymal cells were grown for two weeks from lung minces. At this time, all cells expressed collagen 1 and were designated lung mesenchymal cells. In some experiments, mesenchymal cells were magnetically sorted for expression of CD45 to obtain $\mathrm{CD}_{4} 5^{+}$collagen $1^{+}$fibrocytes or $\mathrm{CD} 45^{-}$collagen $1^{+}$ fibroblasts as previously described. ${ }^{24}$ Briefly, labeled cells were sorted using LS-positive selection columns using a SuperMacs apparatus (Miltenyi Biotech) according to manufacturer's instructions. For extra purity, CD45 + cells were sometimes reapplied to a second LS-positive selection column. For experiments where fibrocyte supernatants were collected, purified fibrocytes were cultured overnight in serum-free media (SFM) with or without anti-periostin Ab (AF-2955, R\&D systems). These cell-free supernatants were then added to purified fibroblasts.

Adoptive transfer. Fibrocytes were purified by magnetic separation from lung mince cultures from untreated wild-type or periostin ${ }^{-1-}$ mice as described above. $5 \times 10^{5}$ fibrocytes were injected via tail vein in a 200 - $\mu$ l volume into mice that had received bleomycin inoculation intratracheally $4 \mathrm{~d}$ previously. Mice were harvested and lungs collected on day 21 after bleomycin, and lung collagen content was determined by hydroxyproline assay.

Flow cytometry. Lung mesenchymal cells were grown for two weeks from lung minces from saline and bleomycin-treated wild-type or periostin $^{-1-}$ mice as previously described. On day 14 cells were collected and incubated with Fc block (1:100) clone 24G2 (BD Pharmingen, San Diego, CA) for $15 \mathrm{~min}$, then stained with combinations of anti-mouse CD45 (BD Pharmingen), anti-mouse CD29 (Beta 1), anti-mouse CD49a (Alpha 1) and anti-mouse CD51 directlyconjugated antibodies available from Biolegend. Cells were run on a FACS Canto (BD Biosciences, Mountain View, CA) and further analyzed using the Flowjo single cell analysis software.

Western blot analysis. Mesenchymal cells were lysed in radioimmunoprecipitation assay buffer with protease inhibitor cocktail [Sigma] for $15 \mathrm{~min}$ at $4{ }^{\circ} \mathrm{C}$ and centrifuged at $10,000 \mathrm{~g}$ for $10 \mathrm{~min}$. Total protein concentrations were determined by the Bicinchoninic acid assay (Pierce). Equal amounts of protein from each sample were separated on a $4-20 \%$ gradient SDS-polyacrylamide gel and transferred to a PVDF membrane (Amersham/GE Healthcare, Pittsburgh, PA). PVDF membrane was probed for alpha smooth muscle actin $(\alpha$-SMA) (Sigma), beta1 integrin (ab52971 Abcam) and rabbit polyclonal glyceraldehyde-3-phosphate dehydrogenase (GAPDH) (Cell signaling, Beverly, MA) as a loading control. Densitometry analyses of Western blot pixel densities were performed using the NIH Image J program.

Statistical analysis. Statistical significance was assessed by analysis of variance (3 or more comparisons) with a Bonferroni post-hoc test or Student $t$ test (2 comparisons) using GraphPad Prism 6 software (San Diego, CA). Data shown represents mean \pm s.e.m., $P<0.05$ was considered significant.

SUPPLEMENTARY MATERIAL is linked to the online version of the paper at http://www.nature.com/mi

\section{ACKNOWLEDGMENTS}

We thank Amanda Huber for technical assistance and comments on the manuscript. Funding sources; NIH/NHLBI HL115618 (B.B.M), UNCF/ MERCK Dissertation Fellowship (SLA), T32 Training grant Al007413 (SLA), NIH/NHLBI HL108904 (K.K.K).

c 2017 Society for Mucosal Immunology

\section{REFERENCES}

1. Wilson, M.S. \& Wynn, T.A. Pulmonary fibrosis: pathogenesis, etiology and regulation. Mucosal Immunol. 2, 103-121 (2009).

2. King, T.E. Jr., Pardo, A. \& Selman, M. Idiopathic pulmonary fibrosis. Lancet 378, 1949-1961 (2011).

3. Hutchinson, J.P., McKeever, T.M., Fogarty, A.W., Navaratnam, V. \& Hubbard, R.B. Increasing global mortality from idiopathic pulmonary fibrosis in the twenty-first century. Ann. Am. Thorac. Soc. 11, 1176-1185 (2014).

4. Martinez, F.J. et al. The clinical course of patients with idiopathic pulmonary fibrosis. Ann. Intern. Med. 142, 963-967 (2005).

5. Antoniou, K.M., Pataka, A., Bouros, D. \& Siafakas, N.M. Pathogenetic pathways and novel pharmacotherapeutic targets in idiopathic pulmonary fibrosis. Pulm. Pharmacol. Ther. 20, 453-461 (2007).

6. Fernandez, I.E. \& Eickelberg, O. New cellular and molecular mechanisms of lung injury and fibrosis in idiopathic pulmonary fibrosis. Lancet $\mathbf{3 8 0}$, 680-688 (2012).

7. Blackwell, T.S. et al. Future directions in idiopathic pulmonary fibrosis research. An NHLBI workshop report. Am. J. Respir. Crit. Care Med. 189, 214-222 (2014).

8. Scotton, C.J. \& Chambers, R.C. Molecular targets in pulmonary fibrosis: the myofibroblast in focus. Chest 132, 1311-1321 (2007).

9. Kalluri, R. \& Neilson, E.G. Epithelial-mesenchymal transition and its implications for fibrosis. J. Clin. Invest. 112, 1776-1784 (2003). 
10. Kalluri, R. \& Weinberg, R.A. The basics of epithelial-mesenchymal transition. J. Clin. Invest. 119, 1420-1428 (2009).

11. Zeisberg, E.M. et al. Endothelial-to-mesenchymal transition contributes to cardiac fibrosis. Nat. Med. 13, 952-961 (2007).

12. Selman, M. \& Pardo, A. Role of epithelial cells in idiopathic pulmonary fibrosis: from innocent targets to serial killers. Proc. Am. Thorac. Soc. 3 , 364-372 (2006).

13. Hashimoto, N. et al. Endothelial-mesenchymal transition in bleomycin-induced pulmonary fibrosis. Am. J. Respir. Cell Mol. Biol. 43, 161-172 (2010).

14. Humphreys, B.D. et al. Fate tracing reveals the pericyte and not epithelial origin of myofibroblasts in kidney fibrosis. Am. J. Pathol. 176, 85-97 (2010).

15. Hung, C. et al. Role of lung pericytes and resident fibroblasts in the pathogenesis of pulmonary fibrosis. Am. J. Respir. Crit. Care Med. 188, 820-830 (2013).

16. LeBleu, V.S. et al. Origin and function of myofibroblasts in kidney fibrosis. Nat. Med. 19, 1047-1053 (2013).

17. Osterholzer, J.J. et al. Implicating exudate macrophages and Ly-6C(high) monocytes in CCR2-dependent lung fibrosis following gene-targeted alveolar injury. J. Immunol. 190, 3447-3457 (2013).

18. Kleaveland, K.R., Moore, B.B. \& Kim, K.K. Paracrine functions of fibrocytes to promote lung fibrosis. Expert Rev. Respir. Med. 8, 163-172 (2014).

19. Bucala, R., Spiegel, L.A., Chesney, J., Hogan, M. \& Cerami, A. Circulating fibrocytes define a new leukocyte subpopulation that mediates tissue repair. Mol. Med. 1, 71-81 (1994).

20. Chesney, J., Bacher, M., Bender, A. \& Bucala, R. The peripheral blood fibrocyte is a potent antigen-presenting cell capable of priming naive Tcells in situ. Proc. Natl. Acad. Sci. USA 94, 6307-6312 (1997).

21. Chesney, J., Metz, C., Stavitsky, A.B., Bacher, M. \& Bucala, R. Regulated production of type I collagen and inflammatory cytokines by peripheral blood fibrocytes. J. Immunol. 160, 419-425 (1998).

22. Moore, B.B. \& Kolb, M. Fibrocytes and progression of fibrotic lung disease. Ready for showtime? Am. J. Respir. Crit. Care Med. 190, 1338-1339 (2014).

23. Moore, B.B. et al. CCR2-mediated recruitment of fibrocytes to the alveolar space after fibrotic injury. Am. J. Pathol. 166, 675-684 (2005).

24. Moore, B.B., Murray, L., Das, A., Wilke, C.A., Herrygers, A.B. \& Toews, G.B. The role of CCL12 in the recruitment of fibrocytes and lung fibrosis. Am. J. Respir. Cell Mol. Biol. 35, 175-181 (2006).

25. Phillips, R.J. et al. Circulating fibrocytes traffic to the lungs in response to CXCL12 and mediate fibrosis. J. Clin. Invest. 114, 438-446 (2004).

26. Madsen, D.H. et al. The non-phagocytic route of collagen uptake: a distinct degradation pathway. J. Biol. Chem. 286, 26996-27010 (2011).

27. Bianchetti, L., Barczyk, M., Cardoso, J., Schmidt, M., Bellini, A. \& Mattoli, S. Extracellular matrix remodelling properties of human fibrocytes. J. Cell. Mol. Med. 16, 483-495 (2012).

28. Kleaveland, K.R. et al. Fibrocytes are not an essential source of type I collagen during lung fibrosis. J. Immunol. 193, 5229-5239 (2014).

29. Xu, J. \& Kisseleva, T. Bone marrow-derived fibrocytes contribute to liver fibrosis. Exp. Biol. Med. (Maywood) 240, 691-700 (2015).

30. Xu, J., Cong, M., Park, T.J., Scholten, D., Brenner, D.A. \& Kisseleva, T. Contribution of bone marrow-derived fibrocytes to liver fibrosis. Hepatobiliary Surg. Nutr. 4, 34-47 (2015).

31. Garcia de Alba, C. et al. Fibrocytes contribute to inflammation and fibrosis in chronic hypersensitivity pneumonitis through paracrine effects. Am. J. Respir. Crit. Care Med. 191, 427-436 (2015).

32. Madala, S.K., Edukulla, R., Schmidt, S., Davidson, C., Ikegami, M. \& Hardie, W.D. Bone marrow-derived stromal cells are invasive and hyperproliferative and alter transforming growth factor-alpha-induced pulmonary fibrosis. Am. J. Respir. Cell Mol. Biol. 50, 777-786 (2014).
33. Naik, P.K. et al. Periostin promotes fibrosis and predicts progression in patients with idiopathic pulmonary fibrosis. Am. J. Physiol. Lung Cell Mol. Physiol. 303, L1046-L1056 (2012).

34. Kudo, A. Periostin in fibrillogenesis for tissue regeneration: periostin actions inside and outside the cell. Cell Mol. Life Sci. 68, 3201-3207 (2011).

35. Horiuchi, K. et al. Identification and characterization of a novel protein, periostin, with restricted expression to periosteum and periodontal ligament and increased expression by transforming growth factor beta. J. Bone. Miner. Res. 14, 1239-1249 (1999).

36. Ruan, K., Bao, S. \& Ouyang, G. The multifaceted role of periostin in tumorigenesis. Cell. Mol. Life Sci. 66, 2219-2230 (2009).

37. Okamoto, M. et al. Periostin, a matrix protein, is a novel biomarker for idiopathic interstitial pneumonias. Eur. Respir. J. 37, 1119-1127 (2011).

38. Izuhara, K. et al. Roles of Periostin in Respiratory Disorders. Am. J. Respir. Crit. Care. Med. 193, 949-956 (2016).

39. Maatta, A., Ekholm, E. \& Penttinen, R.P. Effect of the 3'-untranslated region on the expression levels and mRNA stability of alpha $1(I)$ collagen gene. Biochim. Biophys. Acta 1260, 294-300 (1995).

40. Bonner, J.C. Mesenchymal cell survival in airway and interstitial pulmonary fibrosis. Fibrogenesis Tissue Repair 3, 15 (2010).

41. Hinz, B., Phan, S.H., Thannickal, V.J., Galli, A., Bochaton-Piallat, M.L. \& Gabbiani, G. The myofibroblast: one function, multiple origins. Am. J. Pathol. 170, 1807-1816 (2007).

42. Phan, S.H. The myofibroblast in pulmonary fibrosis. Chest 122, 286S-289S (2002).

43. Uchida, M. et al. Periostin, a matricellular protein, plays a role in the induction of chemokines in pulmonary fibrosis. Am. J. Respir. Cell Mol. Biol. 46, 677-686 (2012).

44. Abe, R., Donnelly, S.C., Peng, T., Bucala, R. \& Metz, C.N. Peripheral blood fibrocytes: differentiation pathway and migration to wound sites. J. Immunol. 166, 7556-7562 (2001).

45. Schmidt, M., Sun, G., Stacey, M.A., Mori, L. \& Mattoli, S. Identification of circulating fibrocytes as precursors of bronchial myofibroblasts in asthma. J. Immunol. 171, 380-389 (2003).

46. Ajayi, I.O. et al. X-linked inhibitor of apoptosis regulates lung fibroblast resistance to Fas-mediated apoptosis. Am. J. Respir. Cell Mol. Biol. 49, 86-95 (2013).

47. Ashley, S.L. et al. Targeting Inhibitor of Apoptosis Proteins Protects from Bleomycin-induced Lung Fibrosis. Am. J. Respir. Cell Mol. Biol. 54, 482-492 (2015)

48. Leask, A. Potential therapeutic targets for cardiac fibrosis: TGFbeta, angiotensin, endothelin, CCN2, and PDGF, partners in fibroblast activation. Circ. Res. 106, 1675-1680 (2010).

49. Lipson, K.E., Wong, C., Teng, Y. \& Spong, S. CTGF is a central mediator of tissue remodeling and fibrosis and its inhibition can reverse the process of fibrosis. Fibrogenesis Tissue Repair 5, S24 (2012).

50. Yang, J., Velikoff, M., Canalis, E., Horowitz, J.C. \& Kim, K.K. Activated alveolar epithelial cells initiate fibrosis through autocrine and paracrine secretion of connective tissue growth factor. Am. J. Physiol. Lung Cell. Mol. Physiol. 306, L786-L796 (2014).

51. Maruhashi, T., Kii, I., Saito, M. \& Kudo, A. Interaction between periostin and BMP-1 promotes proteolytic activation of lysyl oxidase. J. Biol. Chem. 285, 13294-13303 (2010).

52. Reed, N. I. et al. The alphavbeta1 integrin plays a critical in vivo role in tissue fibrosis. Sci. Transl. Med. 7, 288 ra279 (2015).

53. Moore, B.B. et al. Protection from pulmonary fibrosis in the absence of CCR2 signaling. J. Immunol. 167, 4368-4377 (2001).

54. Ashley, S.L., Jegal, Y., Moore, T.A., van Dyk, L.F., Laouar, Y. \& Moore, B.B. gamma-Herpes virus-68, but not Pseudomonas aeruginosa or influenza A (H1N1), exacerbates established murine lung fibrosis. Am. J. Physiol. Lung Cell. Mol. Physiol. 307, L219-L230 (2014). 